\title{
The dental calculus metabolome in modern and historic samples
}

\author{
Irina M. Velsko ${ }^{1,14} \cdot$ Katherine A. Overmyer $^{2}$ - Camilla Speller ${ }^{3} \cdot$ Lauren Klaus $^{4} \cdot$ Matthew J. Collins $^{3,5}$. \\ Louise Loe $^{6}$ - Laurent A. F. Frantz ${ }^{1,7}$ - Krithivasan Sankaranarayanan ${ }^{8}$. Cecil M. Lewis Jr. ${ }^{9}$. \\ Juan Bautista Rodriguez Martinez ${ }^{10} \cdot$ Eros Chaves $^{4,15} \cdot$ Joshua J. Coon $^{2,11,12} \cdot$ Greger Larson $^{1}$. \\ Christina Warinner ${ }^{4,9,13}$ (B)
}

Received: 10 May 2017 / Accepted: 21 September 2017 / Published online: 3 October 2017

(c) The Author(s) 2017. This article is an open access publication

\begin{abstract}
Introduction Dental calculus is a mineralized microbial dental plaque biofilm that forms throughout life by precipitation of salivary calcium salts. Successive cycles of dental plaque growth and calcification make it an unusually wellpreserved, long-term record of host-microbial interaction in the archaeological record. Recent studies have confirmed the survival of authentic ancient DNA and proteins within historic and prehistoric dental calculus, making it a promising substrate for investigating oral microbiome evolution via
\end{abstract}

Data availability: All data generated or analyzed during this study are included in this published article (and its supplementary information files).

Electronic supplementary material The online version of this article (doi:10.1007/s11306-017-1270-3) contains supplementary material, which is available to authorized users.

Christina Warinner

warinner@shh.mpg.de

1 The Palaeogenomics and Bio-Archaeology Research Network, Research Laboratory for Archaeology and the History of Art, University of Oxford, Oxford OX1 3QY, UK

2 Genome Center of Wisconsin, University of Wisconsin-Madison, Madison, WI 53706, USA

3 BioArCh, Department of Archaeology, University of York, York YO10 5DD, UK

4 Department of Periodontics, University of Oklahoma Health Sciences Center, Oklahoma, OK, USA

5 Museum of Natural History, University of Copenhagen, Copenhagen, Denmark

6 Heritage Burial Services, Oxford Archaeology, Oxford, UK

7 School of Biological and Chemical Sciences, Queen Mary University of London, London E1 4NS, UK direct measurement and comparison of modern and ancient specimens.

Objective We present the first comprehensive characterization of the human dental calculus metabolome using a multi-platform approach.

Methods Ultra performance liquid chromatography-tandem mass spectrometry (UPLC-MS/MS) quantified 285 metabolites in modern and historic (200 years old) dental calculus, including metabolites of drug and dietary origin. A subset of historic samples was additionally analyzed by high-resolution gas chromatography-MS (GC-MS) and UPLC-MS/MS for further characterization of metabolites and lipids. Metabolite profiles of modern and historic calculus were compared to identify patterns of persistence and loss.

8 Department of Microbiology and Plant Biology, University of Oklahoma, Norman, OK 73019, USA

9 Department of Anthropology, University of Oklahoma, Norman, OK 73019, USA

10 Dental Office Dr. Juan Bautista Rodriguez, Pozo Alcon, Jaén, Spain

11 Departments of Chemistry and Biomolecular Chemistry, University of Wisconsin-Madison, Madison, WI 53706, USA

12 Morgridge Institute for Research, Madison, WI 53706, USA

13 Department of Archaeogenetics, Max Planck Institute for the Science of Human History, Kahlaische Strasse 10, 07743 Jena, Germany

14 Present Address: Department of Biological Sciences, Clemson University, Clemson, SC 29634, USA

15 Present Address: Pinellas Dental Specialties, Largo, FL 33776, USA 
Results Dipeptides, free amino acids, free nucleotides, and carbohydrates substantially decrease in abundance and ubiquity in archaeological samples, with some exceptions. Lipids generally persist, and saturated and mono-unsaturated medium and long chain fatty acids appear to be well-preserved, while metabolic derivatives related to oxidation and chemical degradation are found at higher levels in archaeological dental calculus than fresh samples.

Conclusions The results of this study indicate that certain metabolite classes have higher potential for recovery over long time scales and may serve as appropriate targets for oral microbiome evolutionary studies.

Keywords Metabolomics $\cdot$ Dental plaque $\cdot$ Oral microbiome $\cdot$ Archaeology $\cdot$ GC-MS $\cdot$ UPLC-MS/MS

\section{Introduction}

Metabolites are small molecular weight molecules produced by a diverse range of enzymatic and chemical reactions, and include products derived from both endogenous and exogenous sources. Profiling metabolites in biological systems to define a metabolome is increasingly common, as it can provide insight into normal and perturbed metabolic processes and their relation to health and disease. Easily obtained human biofluids including serum (Psychogios et al. 2011), urine (Bouatra et al. 2013), and saliva (Dame et al. 2015) have been extensively profiled to define the range of metabolites that are produced in health, and how their levels fluctuate based on changes in activity (Daskalaki et al. 2015), diet (Lankinen et al. 2014), drug use (Fleet et al. 2008; Hahn et al. 1972), and disease progression (Yan et al. 2008). These studies have made it possible to search for specific metabolites that can act as biomarkers for a diverse range of disorders and diseases, including cardiovascular disease (Jensen et al. 2014), diabetes (Sysi-Aho et al. 2011), periodontal disease (Barnes et al. 2009, 2011), and cancer (Beger 2013).

Saliva has become an increasingly popular source for metabolite analysis because collection is simple, non-invasive, does not require training, and it is abundant and easy to resample and store (Dame et al. 2015). Saliva is composed mainly of water, but it also contains a wealth of molecules including mucins, proteins, carbohydrates, salts, and metabolites derived from serum, local cellular processes, diet, and oral microbes (Zhang et al. 2012). Because of the presence of serum-derived molecules, saliva has been used to search for biomarkers both of local diseases, such as periodontal disease (Barnes et al. 2011) and oral cancer (Yan et al. 2008), and also systemic diseases such as pancreatic and breast cancers (Sugimoto et al. 2010), and cardiovascular disease (Foley et al. 2012).
Metabolites from dental plaque, a microbial biofilm that develops on teeth, may provide novel information regarding host-microbiome interactions in health and disease. Dental plaque is likely to contain host saliva- and gingival crevicular fluid (GCF)-derived metabolites in addition to microbial metabolites, potentially providing a substrate for direct comparison of host and microbial activities. For reasons not well understood, dental plaque periodically and rapidly mineralizes to form dental calculus, a substance with concrete-like hardness that is immediately recolonized by oral bacteria in a repetitive process (White 1991). Such rapid entombment has the potential to trap biomolecules from GCF and saliva as well as oral plaque and dietary and environmental debris (Warinner 2016; Warinner et al. 2015).

Although generally kept to low levels by professional dental hygiene regimens today, dental calculus was ubiquitous and relatively abundant in past human populations, as attested by dental calculus preserved within archaeological and paleontological collections spanning tens of thousands of years, and it is also found on the dentitions of some animal species (Warinner 2016; Warinner et al. 2015). Recent biomolecular investigations of ancient dental calculus have demonstrated that it contains exceptionally well preserved DNA and proteins from oral biofilm species, dietary components, and the host (Warinner et al. 2014), as well as preserved plant microfossils (e.g., pollen, starch granules) and metabolic products (e.g., terpenoids) likely originating from dietary and craft activities (Blatt et al. 2011; Buckley et al. 2014; Hardy et al. 2012; Radini et al. 2016; Warinner et al. 2014). Such samples allow deep-time genetic and non-genetic molecular anthropology approaches to studying changes in human behavior, evolution of the oral biofilm and disease processes, and co-evolution of the oral microbiome and host, which are difficult to study using current in vitro and in vivo technologies alone. Gas-chromatography analyses of dental calculus from Neanderthals (Buckley et al. 2014; Radini et al. 2016), pre-agriculturalists (Hardy et al. 2016), and early agriculturalists (Hardy et al. 2012) have been used to infer the use of dietary and/or medicinal plants; however, to our knowledge, no broad-scale analysis to determine the potential range of metabolites trapped in dental calculus has been undertaken.

Here we present an in-depth, metabolic analysis of a set of historic and modern dental calculus samples using a combination of targeted and untargeted approaches to assess the range of metabolites that can be extracted from calculus, and how well they persist and preserve over time. We validated our results and performed targeted metabolite searches in a subset of historic samples for a more thorough assessment of the potential preservation of metabolites of interest. 


\section{Materials and methods}

\subsection{Calculus collection and preparation}

Fresh dental calculus samples were obtained during routine dental cleaning at the University of Oklahoma Periodontology Clinic $(n=1)$ in Oklahoma City, Oklahoma, USA and at a private dentistry practice $(n=4)$ in Jaen, Spain (Table 1). Samples were collected by dental professionals using a dental scaler following standard calculus removal procedures. All samples were obtained under informed consent, and this research was reviewed and approved by the University of Oklahoma Health Sciences Center Institutional Review Board (IRB\# 4543).

Historic dental calculus (Figure S1a) was collected from 12 skeletons in the Radcliffe Infirmary Burial Ground collection, housed at Oxford Archaeology in Oxford, UK (Table 1). This cemetery was used from 1770-c.1855, and the skeletons are not personally identifiable. The oral health of each skeleton was recorded with reference to the presence or absence of caries and periodontal disease, with reference to (Hillson 1996; Ogden 2005). The sex and approximate age at death for each skeleton was estimated following standard osteological criteria (Brooks and Suchey 1990; Buckberry and Chamberlain 2002; Ferembach et al. 1980; Lovejoy et al. 1985; Phenice 1969; Schwartz 1996) and is presented in Table 1. Genetic sex was further confirmed through high-throughput sequencing (HTS) of DNA extracted from additional calculus fragments (described below) following previously described methods (Frantz et al. 2016; Skoglund et al. 2015, 2013); genetic sex determinations for all twelve samples were consistent with those made using osteological approaches. For details see Supplemental Methods.

After collection, the fresh and historic dental calculus samples were stored frozen and transferred on dry ice to Metabolon, Inc. (Durham, NC) for sample processing and metabolite extraction and detection by UPLC-MS/MS. A subset of five historic dental calculus samples (CS6, CS12, CS18, CS24, and CS46) were additionally analyzed at the Departments of Chemistry and Biomolecular Chemistry at the University of Wisconsin (Madison, USA) to further characterize metabolites and lipids by high-resolution gas chromatography (GC)-MS and UPLC-MS/MS (Table 1).
Table 1 Summary of sample demographic and health information and analyses performed

\begin{tabular}{|c|c|c|c|c|c|c|}
\hline ID & Age $^{a}$ & $\operatorname{Sex}^{\mathrm{b}}$ & Smoker & Oral health & Sampled tooth & Analyses performed \\
\hline \multicolumn{7}{|l|}{ Modern } \\
\hline OK1010 & 40 & M & Former & $+\mathrm{PD}$ & - Caries, + PD & UPLC-MS/MS \\
\hline ES28 & 31 & $\mathrm{~F}$ & Current & + Caries, + GV & - Caries, -PD & UPLC-MS/MS \\
\hline ES29 & 30 & M & No & + Caries, + GV & - Caries, -PD & UPLC-MS/MS \\
\hline ES20 & 23 & M & Current & + Caries, + GV & - Caries, -PD & UPLC-MS/MS \\
\hline ES15 & 71 & M & Former & + Caries, + PD & - Caries, +PD & UPLC-MS/MS \\
\hline \multicolumn{7}{|l|}{ Historic } \\
\hline CS06 & $36-45$ & M & N/A & + Caries, +PD & - Caries, -PD & UPLC-MS/ MS ${ }^{\mathrm{c}}$; GC-MS \\
\hline $\mathrm{CS} 12$ & $36-45$ & M & N/A & + Caries, +PD & - Caries, -PD & UPLC-MS/MS ${ }^{\mathrm{c}}$; GC-MS \\
\hline CS18 & $36-45$ & M & N/A & - Caries, +PD & - Caries, -PD & UPLC-MS/MS ${ }^{\mathrm{c}} ; \mathrm{GC}-\mathrm{MS}$ \\
\hline $\mathrm{CS} 24$ & $36-45$ & $\mathrm{~F}$ & N/A & + Caries, + PD & + Caries, + PD & UPLC-MS/MS ${ }^{\mathrm{c}}$;GC-MS \\
\hline CS46 & $>45$ & $\mathrm{~F}$ & N/A & + Caries, + PD & - Caries, -PD & UPLC-MS/MS ${ }^{\mathrm{c}}$; GC-MS \\
\hline $\mathrm{CS} 20$ & $36-45$ & M & N/A & + Caries, + PD & - Caries, -PD & UPLC-MS/MS \\
\hline $\mathrm{CS} 21$ & $26-35$ & M & N/A & - Caries, -PD & - Caries, -PD & UPLC-MS/MS \\
\hline $\mathrm{CS} 23$ & $26-35$ & $\mathrm{~F}$ & N/A & + Caries, +PD & - Caries, -PD & UPLC-MS/MS \\
\hline CS30 & $18-25$ & $\mathrm{~F}$ & N/A & + Caries, - PD & - Caries, -PD & UPLC-MS/MS \\
\hline CS31 & $36-45$ & $\mathrm{~F}$ & N/A & + caries,$+\mathrm{PD}$ & - Caries, -PD & UPLC-MS/MS \\
\hline CS39 & $36-45$ & M & N/A & - Caries, +PD & - Caries, -PD & UPLC-MS/MS \\
\hline CS40 & $>45$ & $\mathrm{~F}$ & N/A & - Caries, -PD & - Caries, -PD & UPLC-MS/MS \\
\hline
\end{tabular}

$P D$ periodontal disease, $G V$ gingivitis, $N / A$ not available, + present, - absent

${ }^{\mathrm{a}} \mathrm{Age}$ of skeletons used for historical calculus sampling is based on osteological indicators and is a range estimate

${ }^{\mathrm{b}}$ Historical skeleton sex estimate is based on both osteological indicators and DNA sequencing, and was in concurrence between the two methods

${ }^{\mathrm{c}}$ UPLC-MS/MS was performed twice; the first at Metabolon, Inc. and the second at the University of Wisconson-Madison 


\subsection{Genetic authentication of a preserved oral microbiome in historic samples}

DNA extracted from a separate fragment of each piece of historic calculus was used to assess microbial community composition. DNA was extracted as previously described, but omitting phenol-chloroform steps (Warinner et al. 2014), and Illumina shotgun sequenced (for details see Supplemental Methods). The 16S rRNA gene-identified reads were then used to assess microbial community composition at the genus level by closed-reference OTUpicking against the GreenGenes v. 13.8 database using UCLUST (Edgar 2010) in QIIME v. 1.9 (Caporaso et al. 2010). The Bayesian analysis-based program SourceTracker (Knights et al. 2011) was used to estimate the source composition of the microbial community identified by QIIME. Human reads were identified by mapping to GRCh38.p10 (GCF_000001405.36) using bwa (Meyer et al. 2012) with the flags aln -1 16500 -o 2 -n 0.01, duplicate reads were removed, and reads mapping to $\mathrm{X}$ and $\mathrm{Y}$ chromosomes were analyzed for genetic sex determination as described above.

\subsection{Mass spectrometry and data processing for UPLC- MS/MS}

Samples $(\sim 20 \mathrm{mg})$ were decalcified in 0.5M EDTA, centrifuged to pellet debris, and supernatant prepared using the automated MicroLab STAR ${ }^{\circledR}$ system from Hamilton Company. Samples were cleaned and divided into five fractions: two for analysis by two separate reverse phase (RP)/UPLC-MS/MS methods with positive ion mode electrospray ionization (ESI), one for analysis by RP/ UPLC-MS/MS with negative ion mode ESI, one for analysis by HILIC/UPLC-MS/MS with negative ion mode ESI, and one sample was reserved for backup. All methods utilized a Waters ACQUITY ultra-performance liquid chromatography (UPLC) and a Thermo Scientific Q-Exactive high resolution/accurate mass spectrometer interfaced with a heated electrospray ionization (HESI-II) source and Orbitrap mass analyzer operated at 35,000 mass resolution. For details see Supplemental Methods. Raw data were extracted, peak-identified and QC processed using Metabolon's hardware and software. Compounds were identified by comparison to library entries of purified standards or recurrent unknown entities. Peaks were quantified using area-under-the-curve. Each compound was corrected in run-day blocks by registering the medians to equal one (1.00) and normalizing each data point proportionately. For specific details about the hardware, software, and libraries used, please see Supplementary Materials Sect. 2.6.

\subsection{Further characterization of historic calculus by GC-MS and UPLC-MS/MS}

Five historic dental calculus samples were selected to further investigate metabolites and lipids in historic dental calculus samples (Table 1). Following sample pulverization, $15 \mathrm{mg}$ was decalcified with $100 \mathrm{uL}$ of $4 \%$ Formic acid. Samples were incubated at $4{ }^{\circ} \mathrm{C}$ with occasional shaking for 12 days. Next, $75 \mathrm{uL}$ of $1 \mathrm{M}$ ammonium hydroxide was added, then samples were extracted with $350 \mathrm{uL} \mathrm{MeOH}+350 \mathrm{uL}$ Acetonitrile (final 2:2:1 Methanol:Acetonitrile:Water). Extract was split for metabolite (GC-MS) and lipid (LC-MS) analysis and dried down by vacuum centrifugation. For GC-MS analysis molecules were analyzed with electron-impact (EI)-Orbitrap full scan of 50-650 m/z range. Lipid LC-MS analysis was performed on a Water's Acquity UPLC CSH C18 Column $(2.1 \times 100 \mathrm{~mm})$ with a $5 \mathrm{~mm}$ VanGuard PreColumn Mobile coupled to a Q Exactive Focus. Raw files were analyzed using an in-house tool for deconvolution of spectra, quantitation, and identification against in-house and NIST 2014 libraries (GC-MS), or the Thermo Compound Discoverer ${ }^{\mathrm{TM}} 2.0$ application with peak detection, retention time alignment, and gap filling (UPLC-MS/MS). Only peaks ten fold greater than solvent blanks were included in the later analysis. The UPLC-MS/MS data was also processed through the Global Natural Products Social Molecular Networking pipeline (Wang et al. 2016). For details see Supplemental Methods.

\subsection{Data analysis}

Mass normalized data were used for all downstream analyses. Data was normalized as follows: values for each sample were normalized by sample mass utilized for extraction, and each biochemical was then rescaled to set the median equal to 1. Lastly, missing values were imputed with the minimum. First, overall metabolome composition was summarized at the super-pathway, sub-pathway, and metabolite levels, and identified metabolites were cross-referenced against public databases to obtain KEGG compound identifiers and Human Metabolome Database (HMDB) IDs. Next, metabolites found to be ubiquitously present in modern samples and ubiquitously absent in historic calculus were compared. Here ubiquity among the five modern samples was applied as a measure to identify highly prevalent (potentially core) dental calculus metabolites; complete absence of these metabolites among all twelve historic samples was used to identify metabolite candidates that may be particularly prone to loss or that are unstable and susceptible to degradation through time-dependent taphonomic processes. Following this analysis, differential representation of metabolites between historic and modern samples was determined using the program Statistical Analysis of Metagenomic Profiles 
(STAMP) (Parks and Beiko 2010; Parks et al. 2014), first including metabolites detected in both historic and modern samples, and then again using only those metabolites that were universally detected in all 17 calculus samples. Metabolite profiles of historic and modern samples were compared using 2 group analysis of the average quantity of each metabolite and analyzed by White's non-parametric two-sided $t$ test with bootstrapping to determine the difference between proportion (DP) with cut-off $95 \%$ and Storey's FDR. Differential abundance was determined in hierarchical categorization of super-pathway, sub-pathway, and individual metabolite. For both analyses corrected $p$ values ( $q$ values) of $\leq 0.05$ together with an effect size $\geq 1$ were considered significant. Pathway maps were created using iPATH2 (Yamada et al. 2011) for metabolites with KEGG compound identifiers. Partial Least Squares Discriminant Analysis (PLS-DA) was performed using the R package mixOmics (Rohart et al. 2017) in default settings, and Q2 values for the PLS-DAs was calculated in the R package DiscriMiner (https://github.com/gastonstat/DiscriMiner) using 2 components to match the mixOmics calculations, and "leave one out" cross-validation.

\section{Results}

\subsection{Authentication of historic calculus}

Archaeological specimens are subject to environmental degradation and contamination, and thus it is necessary to confirm of the source (e.g., endogenous microbiome vs. exogenous environmental microbes) of biomolecules detected in ancient samples. QIIME and SourceTracker analyses confirmed excellent biomolecular preservation of an in situ oral microbial community within the historic dental calculus samples, and16S rRNA gene sequences closely matched those expected for dental plaque communities, with minimal contamination from exogenous sources such as soil and skin (Figure S1b). The high proportion of microbes of "unknown" source in several historic dental calculus samples is observed in modern calculus samples (Ziesemer et al. 2015) (Fig. 1b 'Modern'), and is a result of mismatched source samples. Several poorly taxonomically characterized oral taxa such as Methanobrevibacter and Tissierellaceae are highly abundant in mature dental calculus biofilms but are infrequently detected in healthy oral plaque early biofilms such as the Human Microbiome Project cohort we used as source samples. Therefore these genera cannot be confidently assigned to an oral plaque source, and are instead attributed to an unknown source.

\subsection{Metabolic pathway coverage in dental calculus}

A total of 285 metabolites were identified by UPLC-MS/ MS in the 17 dental calculus samples, and these were categorized as members of one of eight super-groups: Amino acids, Carbohydrates, Cofactors and vitamins, Energy, Lipids, Nucleotides, Peptides, and Xenobiotics (Fig. 1a; Supplemental Table S1), which were further classified into 69 sub-categories. More than half of the metabolites $(n=185)$ were detected in both historic and modern calculus samples, demonstrating that metabolites can be recovered from historic dental calculus, while 99 metabolites were detected only in modern samples and one was detected only in historic samples. One hundred ninety-nine metabolites were detected in all five modern samples, 97 were detected in all 12 historic samples, and 85 were detected in all 17 calculus samples (Table S1). A smaller subset of historic calculus was analyzed by GC-MS and LC-MS at the University of Wisconsin-Madison; this enabled the identification of 15 additional metabolites and 40 additional lipids, respectively (Fig. 1b; Table S2). For metabolites that were quantified in the main analysis and in the smaller subset, we see comparable quantitation despite differences in extraction procedures and analysis methods between Metabolon and the University of Wisconsin (positive correlation, $\mathrm{R}^{2}=0.7$, Supplemental Figure S2). UPLC-MS/MS-identified metabolites with KEGG compound identifiers $(n=207)$ were located on a general metabolic pathway map (Figure S3) and a map of biosynthesis of secondary metabolites (Figure S4).

To try to characterize more of the unknown features, we analyzed the LC-MS data acquired on the subset of historic calculus through the Global Natural Products Social Molecular Networking (GNPS) pipeline (Wang et al. 2016). This online tool searches experimental MS2 spectra against a large number of publically available spectral libraries and clusters spectra based on shared fragment ions. The identified clusters were primarily molecules that had been identified previously by Metabolon. Expectedly, we found molecular clusters associated with lipid class: for example, phosphatidylcholines clustered within the same molecular network, and these molecules shared network connections with sphingomyelins containing phosphocholine head groups (Figure S5). We also observed clusters of molecules that could be categorized as plasticizers, which had not been identified in the data set by metabolon; however, we suspect these molecules were introduced during sample processing. For example, one of these molecules-didodecyl 3,3'-thiodipropionate - has been previously reported to be leached from polypropylene tubes during organic extractions (Xia et al. 2005). 
A
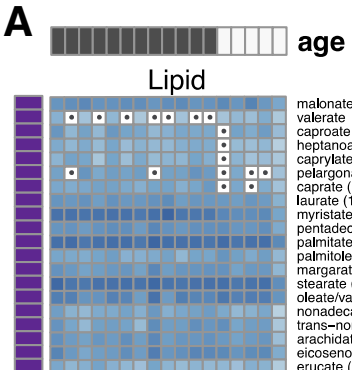

malonate
valerate
caproate (6:0)
heptanoate $(7: 0)$

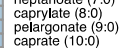

paargonale:
carate (10:0)
luarate (12:0)
myristate (14:0)

myristate (14:0)
pentadecanoate (15:0)
palmitate (16:0)

palmitate $(16: 0)$
palmitoleate $(16: 1 \mathrm{n})$
margarate $(17: 0)$

stearate $(18: 0)$
oleatelvaccenate (18:1)
nonadecanoate (19:0)

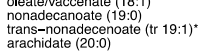

$\because \because \because \because \because \because \because \because \bullet$

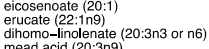

dihomo-linolenate
mead acid (20:3n9)
13 -methylmyristat

13 -methylmyristate
15 -methylyalmitate
17 -methylstearate

17-methylstearate
glutarate (pentanedioate)
2 -hydroxyglutarate

$4-$ hydroxy-2-oxoglutaric acid
adipate

$\rightarrow \cdot$

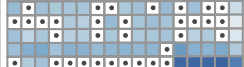

azelate (nonanedioate)
sebacate (decanedioate)
dodecanodioate

dodecanedioate (MMA)
methylmalonate (MMA)

methylmalona
deoxycarnitin
carnitine

$\because \because \because \because \cdots$

$\bullet \quad \because \cdots$

$\bullet \bullet \bullet \bullet \bullet \bullet$

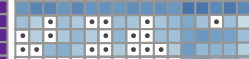

-hydroxybutyrate (BHBA)
2-hydroxydecanoate
2-hydroxypalmitate

2-hydroxystearate
3-hydroxydecanoate

3-hydroxylaurate
3-hydroxymyristate
-hydroxypalmitate

3-hydroxystearate
3-hydroxystearate
palmitoyl ethanolamide

stearoyl ethanolan
choline

hloline phosphate
glycerophosphorylcholine (GPC)
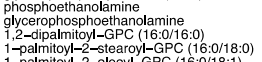

1-palmitoyl-2-oleoyl-GPC (16:0/18:1)
1-palmitoyl-2-linoleopl-GPC (11:0/18:2
1-stearoyl-2-oleoyl-GPC (18:0/18:1)

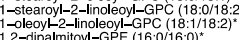

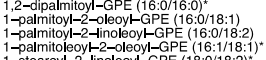

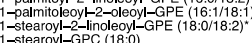

1-palmito-GII-GPE (16:0)
1 -stearoyl-GPE (18:0)

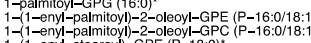

$1-(1-$ enyl-stearoyl)-Ge
glycerol

glycerol 3-phosphate
glycerophosphoglycero

sphinganine
N-palmitoly-sphinganine (d18:0/16:0)
palmitoyl dihydrosphingomyelin (d18:0/16:0)

palmitoyl dihydrosphingomyelin (d18)
palmitoyl sphingomyelin (d118:116:0)
stearoyl sphingomyelin (d18:1/18:0)

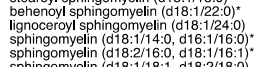

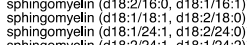

sphingomyelin
sphingosine

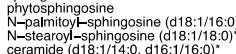

ceramide (d18:1/14:0, d16:1/16:00*
lactosyl-N-palmitoyl-sphingosine (d18:1/16:0)

$\cdot$

cholesterol
7 -ketocholesterol

4-cholesten-3-one
7-hydroxycholesterol (alpha or beta)
dehydroiscandrosterone sulfate (DHEA-S)

dehydroisoandros
cholate
chenodexycholate

$\because \because \div !$

Energy
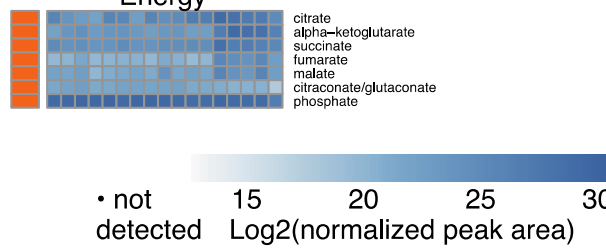

historic

modern

$\because \ldots \ldots$

$\cdot \because: \div: \because \cdot \div: \div$

\section{|IIH|||||||| age}

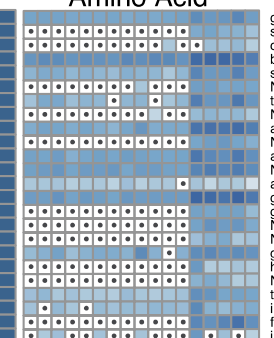

$\because \because \because \div:$

•・・・・・・・・・・・

$\because \because \because \because \because \because \because \because$

$\therefore \therefore$

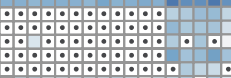

$\cdots \cdots \cdots \cdots$

$\because \cdots \because \because \because \because \because$

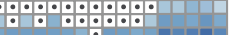

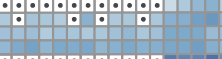

$\because \because \because \div \because \div \div \div$
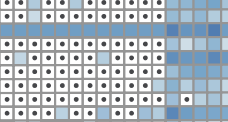

$\because \cdots \cdots \cdots \cdots \bullet \bullet$

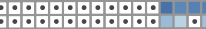

•・・・・・・・・・・・
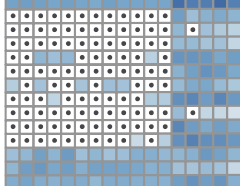

Xenobiotics
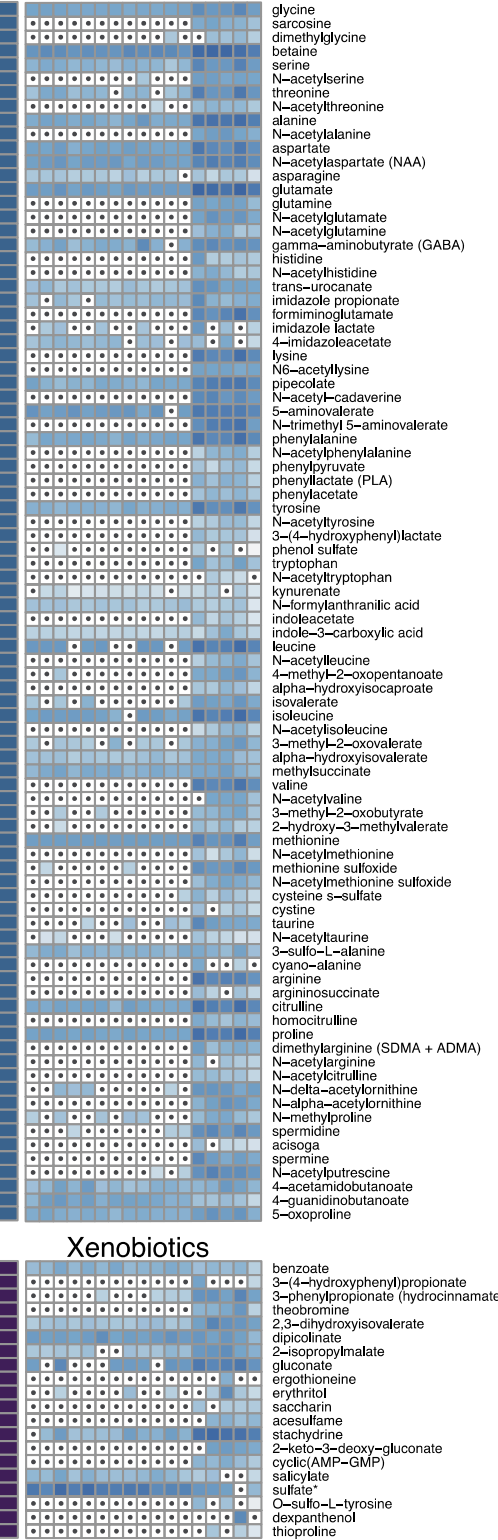

-acetylglutamine
amma-aminobutyrate (GABA)

N-actiotylyistidine
trans-urocanate

midazole propionate
formiminoglutamate

midazole lactate
4-imidazoleacetate

None
N6-acetyllysine
pipecolate

pipecolate
N-acetyl-cadaverine
5 -aminovalerate

N-trimethyl 5-aminova
phenylalanine
N-acetylphenylalanine

phenylyyruvate
phenyllactate (PLA)

phenylacetate
tyrosine

N-acetyltyrosine
3-(4--hydroxypheny)lactate

phenol sulfate
tryptophan

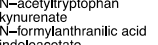

-formylanthranilic acid
ndoleacetate

$\mathrm{N}$
leucine
$\mathrm{N}$-acetylleucine

4-methyll-2-oxine
alpha-hytanoate

isovalerate
isoleucine

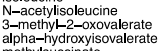

alpha-hydroxyisovale
methylsuccinate

valiny
$\mathrm{N}$-acetylvaline

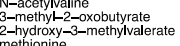

methionine
$\mathrm{N}$-acetylymethionine
methionine sulfoxide

cystein
cystine
taurine

$\mathrm{N}$-acetyltaurine
3-sulfo- $L$-alanine

cyano-alanine
arginine
argininosuccinate

citrulline homocitrulline

proline
dimethylarginine (SDMA + ADMA)
N-acetylarginine

$\mathrm{N}$-acetylyarginine
$\mathrm{N}$-acetylcitrulline

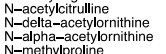

N-methylprol
spermidine

spersidane
acioga
spermine

spermine
N-acetylputrescine
4-acetamidobutanoate

- guanidinobutan
-oxoproline

$B$ |ा age

Subset GC-MS
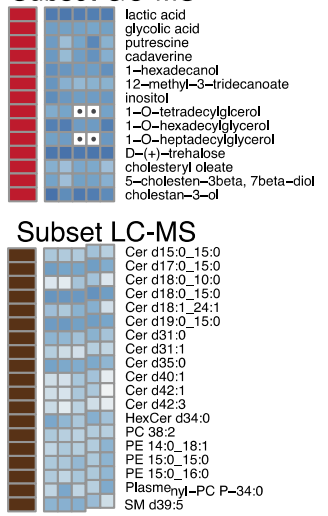

Cofactors and Vitamins

B

SM d39:5
Fig. 1 Heat map summary of metabolites observed in modern and historic dental. Metabolites were quantified by area under the curve and normalized to mass of sample extracted. a UPLC-MS/MSdetected metabolites. Samples were hierarchically clustered, and $\log 2$

\subsection{Comparison of dental calculus and saliva metabolomes}

To determine the degree of overlap between metabolites in dental calculus and metabolites in saliva, we compared
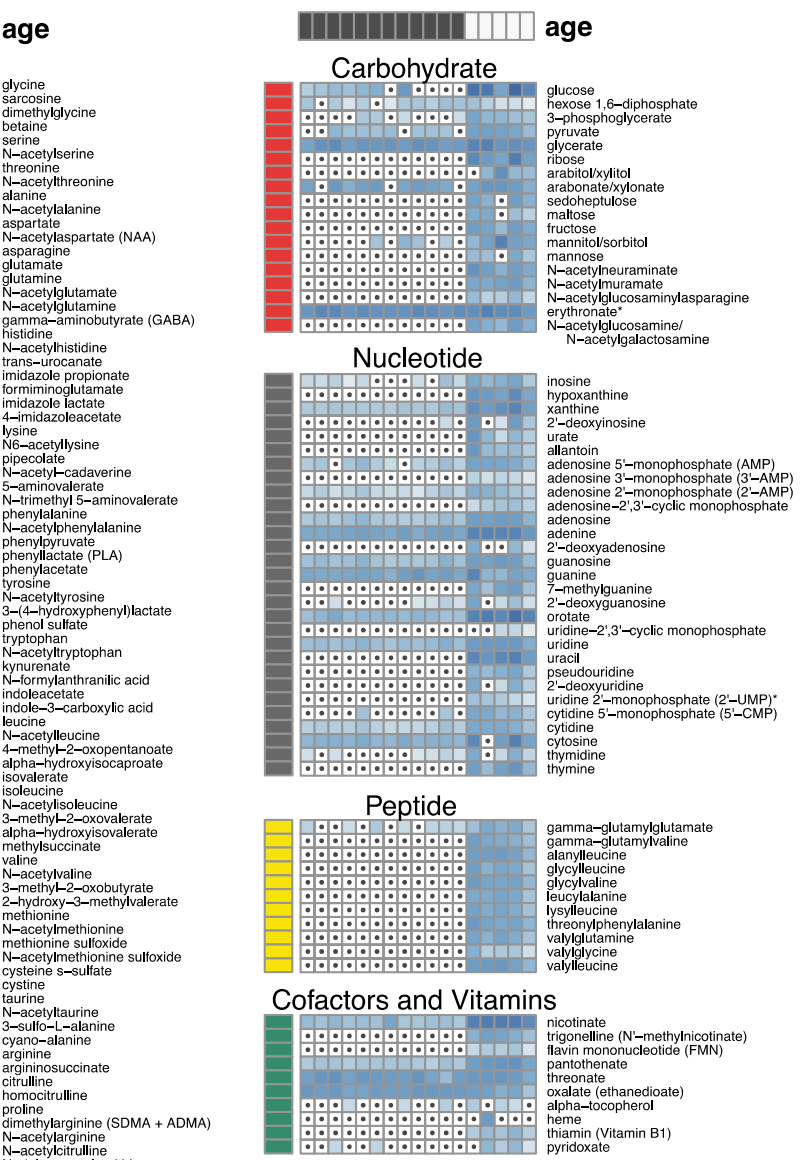

transformed values are presented above, grouped by super-pathway. b Metabolites detected by GC-MS and LC-MS in historic calculus. Non-filled cells containing a dot indicate the compound was not detected

our results to the saliva metabolome. We downloaded a list of all metabolites reported in saliva as catalogued in the Human Metabolome Database (Wishart et al. 2013) version 3.6 (hmdb.ca) as of February 2017 to use as the known saliva metabolome. This list contained 1233 metabolites of 
endogenous and exogenous origin, spanning the full range of super-pathways detected in calculus samples. Just over half, 159 , of the 285 metabolites detected in calculus (55.7\%) were previously included in HMDB's saliva metabolome (Table S1), while these 159 metabolites make up just $12.9 \%$ of the total metabolites detected in saliva. Of the remaining 107 metabolites detected in calculus, 84 have been detected in blood, urine and/or cerebrospinal fluid, and 23 have no HMDB identifier. At least one metabolite in each of the sub-pathways represented in calculus is not included in the HMDB saliva metabolome.

\subsection{Metabolite preservation patterns}

Among metabolites that are likely endogenous (host or oral microbiome) in origin (i.e., not xenobiotics), metabolite persistence differs by super-pathway in historic samples (Fig. 1). Overall, historic samples had lower representation in metabolites categorized as Amino acids, Vitamins and cofactors, Carbohydrates, Nucleotides, and Peptides (Fig. 2a). In contrast, Lipids and Energy metabolites were generally observed in both historic and modern calculus (Fig. 2a). Additionally, at a finer scale, certain chemical configurations appear to be lost through time, for example $\mathrm{N}$-acetylation, amino acids with positively-charged R-groups, and phenyl rings one carbon away from an oxygen. These data suggested a preservation bias that could be due to either chemical stability or compound solubility, although it is a possibility that low sample amounts limit the detection of certain metabolites.

To test the hypothesis that preservation is linked to aqueous solubility, we compared the differential abundance of metabolites between modern and historic calculus to the predicted hydrophobicity of the compounds. The predicted hydrophobicity was extracted from the HMDB, which sources the ALOGPS predicted ratio of compound partitioning between 1-octanol and water $(\log \mathrm{P})$ (Tetko and Bruneau 2004). When plotting fold-change abundance (modern/ historic) to $\log \mathrm{P}$, we observe a significant $(\mathrm{p}<0.001)$ negative correlation (Fig. 2b), suggesting that molecules that are more abundant in modern calculus have lower organic solubility and higher aqueous solubility. This result is consistent with our hypothesis that metabolite preservation is in part due to aqueous solubility. The exception to our hypothesis, and the outlier in Fig. 2b, are poly-unsaturated fatty acids (PUFAs), which have high logP but low preservation. Two PUFAs, mead acid (20:3n9) and dihomo-linolenate (20:3n3 or n6), were identified in all modern dental calculus samples but were not observed in historic samples. The loss of these PUFAs in historic calculus may be partially explained by the decreased oxidative stability of fatty acids with decreasing saturation (Cosgrove et al. 1987; Rustan and Drevon 2005).

As yet there are no detailed assessments of metabolite degradation in archaeological dental calculus; however, analyses of protein damage patterns in human dental
A

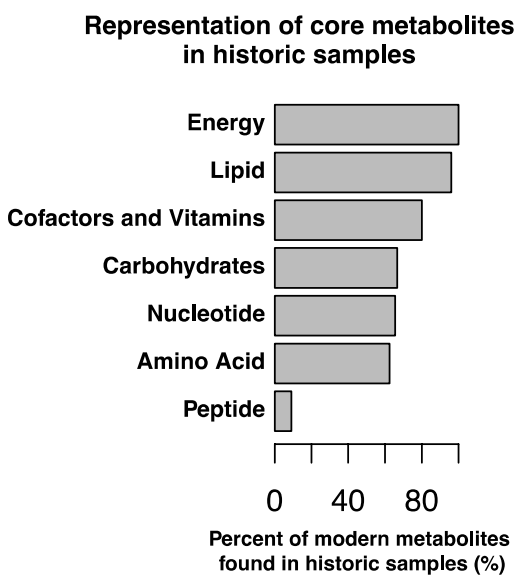

B

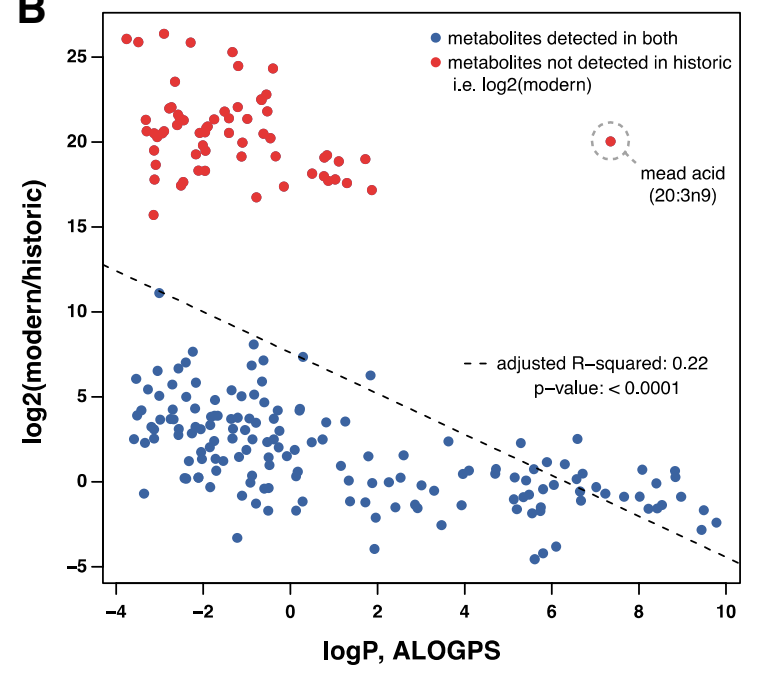

Fig. 2 Compound preservation is correlated with aqueous solubility. a Percent of high ubiquity metabolites in modern calculus that were also recovered in at least one historic calculus sample. Peptides exhibit the poorest representation in historic dental calculus, with only $9 \%$ of the peptides observed to be present in all five modern calculus samples also detected in any historic sample. By contrast, Lipids and Energy (TCA cycle) super-pathways exhibit high representation in historic calculus, with $>96 \%$ of compounds found in all five modern samples also recovered from historic dental calculus. Xenobi- otics, which largely comprise dietary and pharmaceutical compounds, are not shown. b The $\log 2$ fold-change (modern/historic) of metabolite abundance versus the 1-octanol versus water partition coefficient $(\log \mathrm{P})$, estimated with the ALOGPS tool. In the cases where metabolites were only detected in modern calculus, the $\log 2$ values (not foldchange) were plotted relative to $\log$ P. The fitted linear model showed a significant effect $(\mathrm{p}<0.0001)$ of $\log \mathrm{P}$ on metabolite fold-change with and adjusted $\mathrm{R}^{2}$ of 0.22 . The outlier from this trend was mead acid (20:3n9) 
calculus and mammoth bone (Cappellini et al. 2012) can be used to draw comparisons with damage patterns in calculus metabolites. Warinner, et al. (2014) found that the most common protein post-translational modification products in ancient dental calculus are deamidation of asparagine, deamidation of glutamine, oxidation of methionine, and conversion of N-terminal glutamine to pyroglutamate. Asparagine was detected in all five modern samples and 11 historic samples, while the deamidation product aspartate was detected in all 17 samples, and in higher quantity. Glutamine was detected in all modern samples but not in historic samples, and its deamination products glutamate and 5-oxoproline were detected in higher concentration in all 17 samples, although at much higher concentration in modern than historic samples. Oxidation, which is widely documented in the degradation of oil paintings (Oakley et al. 2015) and food spoilage (Velasco and Dobarganes 2002) also occurs in dental calculus. For example, the ratio of cholesterol and its oxidation product 7-ketocholesterol was reversed between modern and historic calculus, and kynurenin, an oxidation product of tryptophan that is known to accumulate in archaeological bone over time (Cappellini et al. 2012), was detected in historic calculus while tryptophan was absent. In contrast, methionine was detected in all 17 samples, and was much more abundant in modern samples, while the oxidation product methionine sulfoxide was detected in all five modern samples and in only two historic samples, in all cases at lower concentrations. Free methionine sulfoxide may be unstable and subject to further rapid breakdown.

\subsection{Lipid 2-hydroxylation as an indicator of calculus age}

Four 2-hydroxylated lipids were detected in all calculus samples-2-hydroxyadipate, 2-hydroxystearate, 2-hydroxypalmitate, and 2-hydroxyglutarate-and the first three are more abundant in ancient than modern samples. The only metabolite detected in historic but not modern samples was 2-hydroxydecanoate (detected in 10 of the 12 historic samples), while the parent molecule decanoate was not detected in any of our samples. In contrast, 3-hydroxylated and 2,3-hydroxylated lipids are more abundant in modern than historic samples. The increased presence of 2-hydroxylated lipids in ancient samples suggests that this modification may increase over time. The difference in patterns of lipid hydroxylation between ancient and modern calculus suggests that in some cases 3-hydroxylation may switch to 2-hydroxylation.

\subsection{Differentially abundant metabolites in ancient and modern calculus}

To further define the differences in metabolic functions preserved through time, we compared the metabolites present in both modern and ancient calculus samples at the super-pathway, sub-pathway, and individual metabolite levels using STAMP. Principal components analysis demonstrated distinct separation of modern and historic samples (Fig. 3a) with tight clustering of historic samples along PC1 and $\mathrm{PC} 2$, while modern samples were more distributed, suggesting that loss of metabolites through time results in a more uniform metabolite profile between samples than may have originally existed. Comparing the mean proportions of individual metabolites detected in at least one modern and one historic sample, 161 were significantly more abundant $(\mathrm{q} \leq 0.05)$ in one sample set, yet only 21 additionally had an effect size of $\geq 1.0$ (Fig. 3b, Table S3 bold metabolites). When considering only the metabolites that were universally present in all five modern and all 12 historic samples (Table S3, superscript 'c'), a slightly different set of metabolites and metabolic pathways are differentially abundant. Historic and modern samples still separate distinctly in PCA (Figure S6a), but many more metabolites have a significant difference in mean proportions (Figure S6b).

We then examined differences in proportion of superpathways, sub-pathways, and individual metabolites present in at least one modern and historic sample to better understand the patterns of preservation and loss through time. The proportion of Lipids was significantly higher in ancient than modern calculus samples, suggesting that non-polar, chemically inert molecules are particularly stable through time (Fig. 4a). On the other hand, the proportion of Amino acids, Carbohydrates, Cofactors and vitamins, and Xenobiotics were significantly higher in modern calculus (Fig. 4a), demonstrating substantial loss and/or degradation of metabolites in these super-pathways over time. The super-pathway Peptides was excluded from analysis using this method because its near total absence in historic samples resulted in very few possible comparisons.

As expected from the super-pathway differential abundances, many of the sub-pathways with greater proportional representation in historic calculus were related to lipid metabolism (Fig. 4b). The sterols include cholesterol and its oxidation products 4-cholesten-3-one, 7-hydroxycholesterol (alpha or beta), and 7-ketocholesterol, which is consistent with the expectation that increased oxidation will occur over time. Guanidino and acetamido metabolism comprised a greater proportion of historic sample metabolites due to overrepresentation of 4-guanidinobutanoate, a product of arginine and putrescine metabolism. Several of the sub-pathways with greater proportional representation in modern samples can be explained by a single metabolite, 
Fig. 3 Differences exist in mean proportions of metabolites detected in at least one historic and one modern dental calculus sample. a Principal components analysis distinctly separates modern and historic calculus samples. b Metabolites with significant differences $(\mathrm{q} \leq 0.05$, effect size of $\geq 1.0$ ) in mean proportions between historic and modern calculus

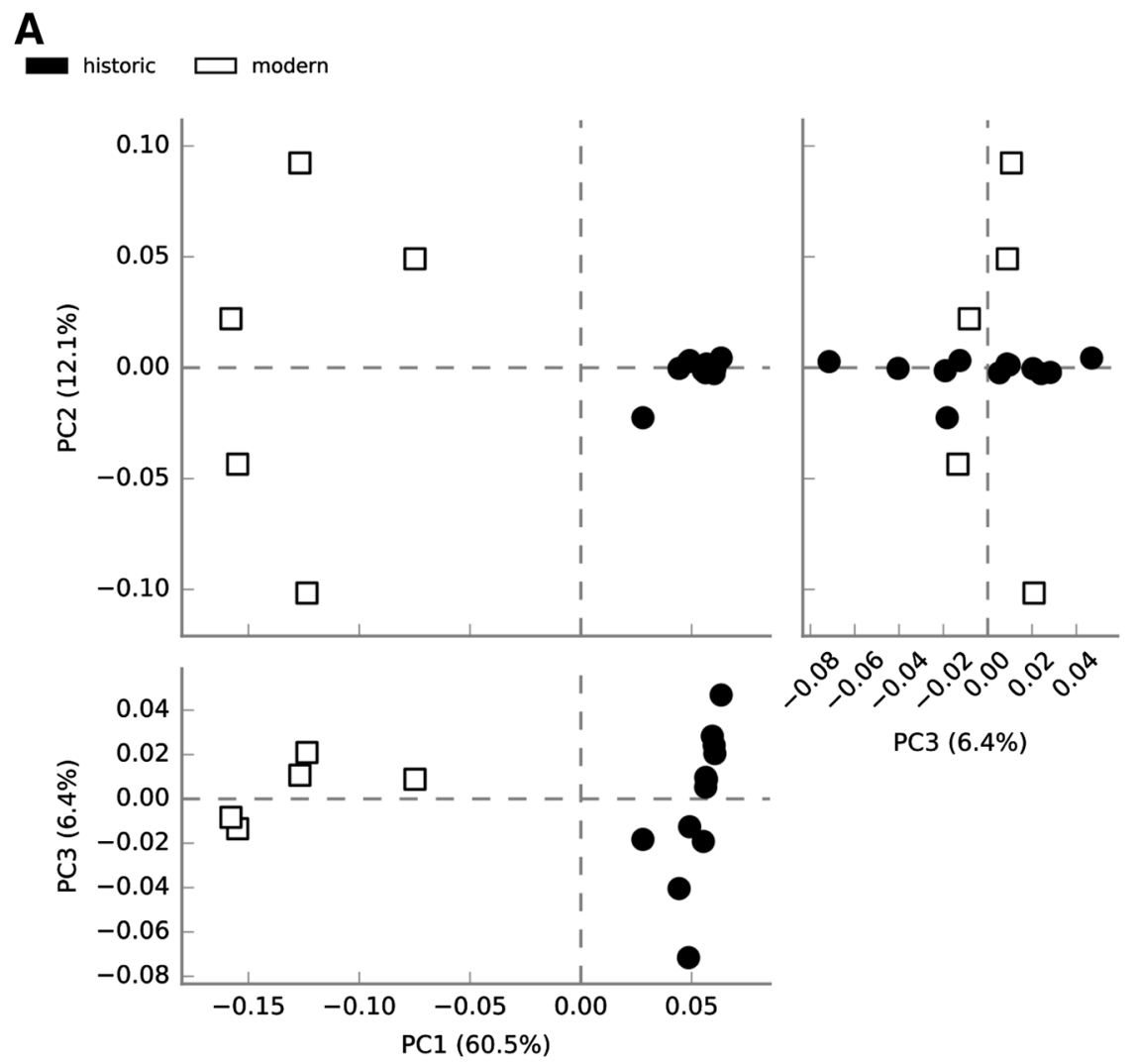

B

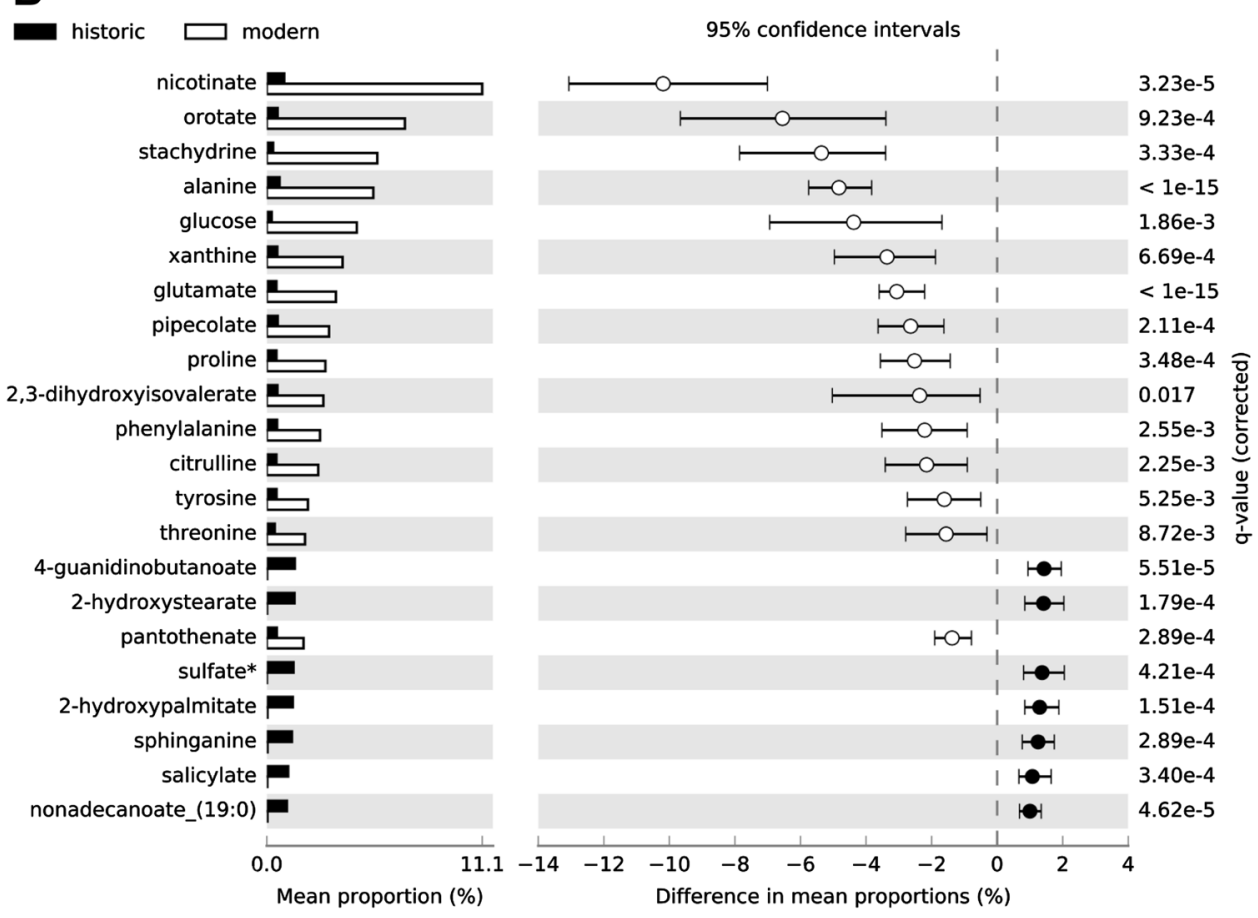

which manifests at the metabolite level (Fig. 4b), and these include Nicotinate and nicotinamide metabolism, Pyrimidine metabolism, and Food component/plant. A single metabolite each comprises the Chemical and Drug sub-pathways, sulfate and salicylate, respectively. With respect to the latter, salicylate is abundant in modern pharmaceuticals, but may have been consumed medicinally in the form of willow bark tea by the historical population, especially given the 
Fig. 4 Differences exist in proportions of super-pathways, sub-pathways, and metabolites represented in at least one historic and one modern dental calculus sample. Significantly different ( $\mathrm{q} \leq 0.05$, effect size of $\geq 1.0$ ) proportions of a Superpathways, b Sub-pathways, and $\mathbf{c}$ metabolites. Individual metabolites between historic and modern samples
A

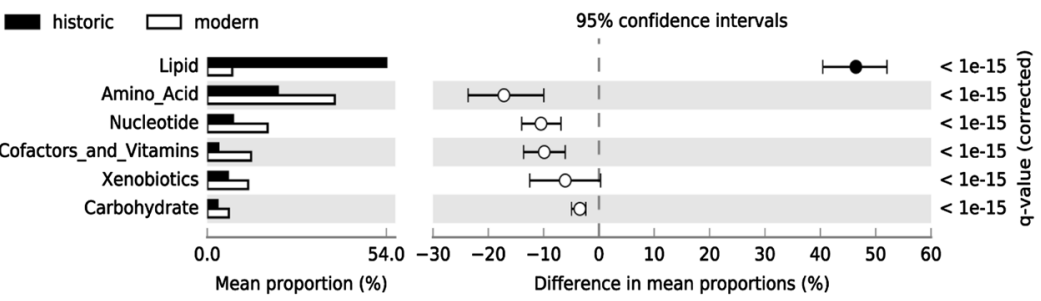

B

historic $\square$ modern

Nicotinate_and_Nicotinamide_Metabolism Food_Component/Plant Long_Chain_Fatty_Acid Sphingolipid_Metabolism Pyrimidine_Metabolism,_Orotate_containing Fatty_Acid,_Monohydroxy Urea_cycle;_Arginine_and_Proline_Metabolism Alanine_and_Aspartate_Metabolism Medium_Chain_Fatty_Acid Purine_Metabolism,_(Hypo)Xanthine/Inosine_containing Fatty_Acid,_Dicarboxylate Glycolysis,_Gluconeogenesis,_and_Pyruvate_Metabolism Phosphatidylcholine_(PC) Lysine_Metabolism Glutamate Metabolism Ceramides

Fatty_Acid,_Branched Phenylalanine_Metabolism Glycine,_Serine_and_Threonine_Metabolism

Tyrosine_Metabolism Guanidino_and_Acetamido_Metabolism Pantothenate_and_CoA_Metabolism $\square$ Chemical

Tryptophan_Metabolism Ascorbate_and_Aldarate_Metabolism $\mathbf{F}$ Drug $\boldsymbol{F}$

95\% confidence intervals

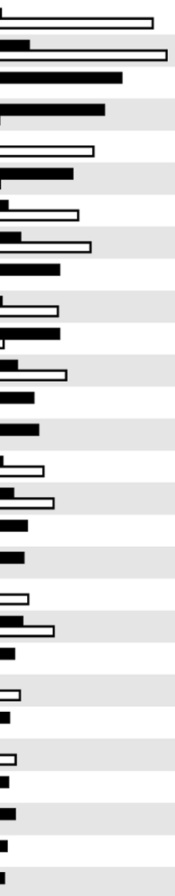

\begin{tabular}{l|lll} 
& & $<-15$
\end{tabular}
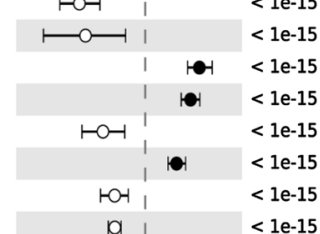

id । $\quad<1 \mathrm{e}-15$

। < $<1$ - 15

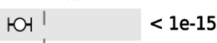

< < 1e-15

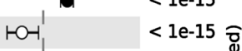

, 1 < 1e-15

< < 1e-15

al $<1 \mathrm{e}-15$

|어 < 1e-15

$<1 \mathrm{e}-15$

- < e-15

어 < le-15

|| < $1 \mathrm{e}-15$

- <1e-15

|al < e-15

< $<1 \mathrm{e}-15$

$0^{\prime}<1 e-15$

- <1e-15

- < 1e-15

$<1 \mathrm{e}-15$

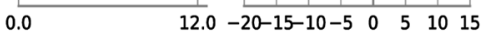

Mean proportion (\%) Difference in mean proportions (\%)

\section{C}

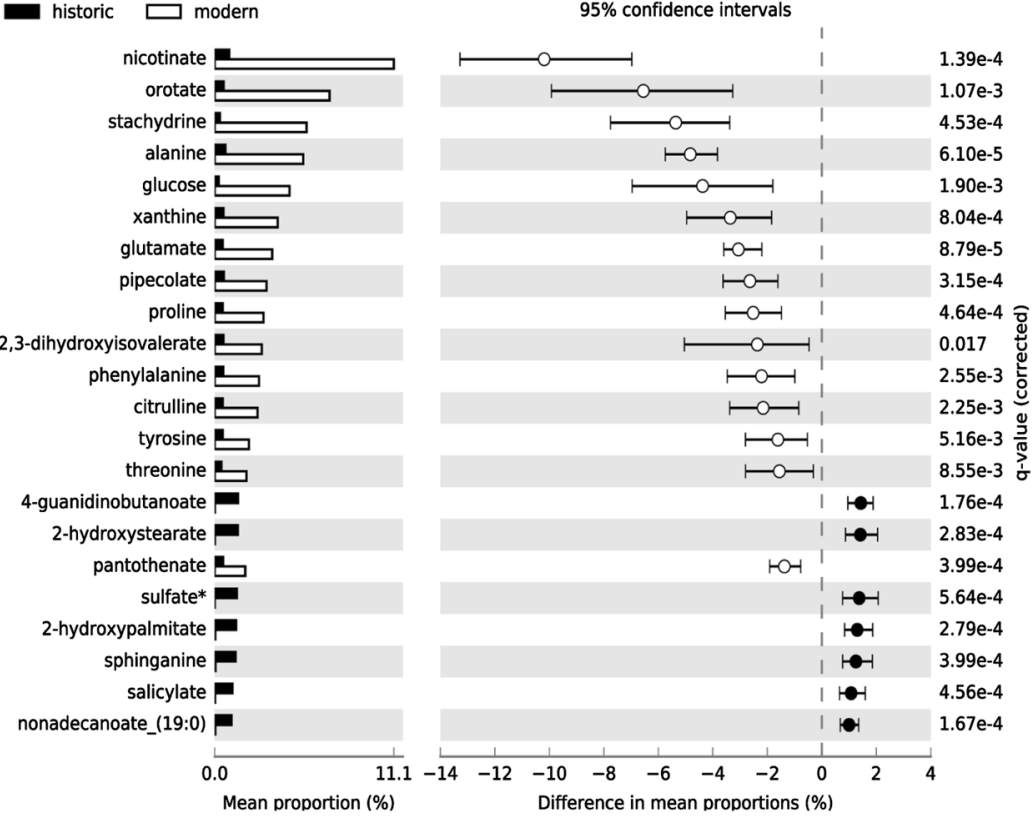


fact that they were buried in a hospital-associated cemetery. Salicylates are also naturally found in a variety of fruits, vegetables, herbs, and spices; however at levels far below the therapeutic doses typical of modern pharmaceuticals (Castillo-García et al. 2015). Alternatively, the salicylates in the historic calculus samples may be a plant root-derived product (Badri and Vivanco 2009), resulting from the diffusion highly soluble, small organic acids into the calculus from burial soil.

In contrast to the large number of sub-pathways representing a significantly higher proportion of metabolites between historic and modern calculus, only 22 individual metabolites were significantly differentially represented between historic and modern calculus (Fig. 4c). Modern calculus had significantly higher proportions of nicotinate, orotate, stachydrine, alanine, and glucose (Fig. 4c). Both nicotinate and orotate may be taken as a dietary supplement, while stachydrine (proline betaine), is a plant metabolite that is not metabolized by mammals (Lever et al. 1994), but is common in citrus fruits and orange juice (Atkinson et al. 2007). Low abundance of stachydrine in historic calculus may relate to dietary differences between these modern and historic populations, or to differential preservation. Alanine, the smallest amino acid, and glucose may be lost through high solubility. The majority of metabolites with significantly greater proportion in historic samples are lipids, including 2-hydroxystearate.

In addition to endogenous metabolites, several xenobiotics found to be present only in modern calculus samples are from food and pharmacologic agents introduced to or popularized in European populations in the twentieth century, including the artificial sweeteners acesulfame, saccharin, and arabitol/xylitol. Additionally, theobromine, an alkyloid present in coffee, tea, and chocolate, was also only detected in modern calculus, suggesting that consumption of these products by the historic population was absent or low, despite their increasing availability in Europe in the 1800s, or that this metabolite poorly preserves over time.

\subsection{Potential for maintenance of biological signatures in calculus metabolite profiles}

Unlike saliva, dental calculus does not represent a snapshot of a specific time and specific metabolic state, but rather it represents a life history in which specific profiles may be diluted out by fluctuating metabolic processes throughout an individual's life, loss of unstable metabolites over time, and random chance with respect to the entrapment of xenobiotics and dietary compounds. However, distinct metabolic signatures related to biological variables such as sex (Takeda et al. 2009), oral health status (Barnes et al. 2009, 2011), and oral biofilm microbial composition (Takahashi et al. 2010) have been reported in saliva, GCF, and oral plaque samples, all of which are likely to contribute to the metabolite profile in dental calculus. Therefore, we assessed differences in metabolic profiles between calculus from different age groups, sex, and oral health status by partial least squares discriminant analysis (PLS-DA) (Q2 valuesTable S4), and, further, looked for metabolites that could be specifically attributed to bacterial activity.

No differences were found in the metabolite profile between age groups, between males and females, between samples from caries-affected and caries-free dentitions, or between samples from periodontal disease-affected and non-affected individuals when considering metabolites detected in at least one historic and one modern calculus sample (Fig. 5a), or metabolites universally present in all 17 samples (Figure S8a). However, there was a distinct separation of modern and historic samples in each comparison so we repeated PLS-DA using only historic sample data. PLS-DA separated historic samples based on sex, age, caries status and periodontal disease status when using metabolites detected in at least one historic sample (Fig. 5b) and when using only metabolites present in all 12 historic samples (Figure S8b). Applying PSLDA to universally detected metabolites in only the Lipid and Energy classes, (the best-preserved classes in historic samples, Fig. 1a) separated samples based on time period rather than biological category (Figure S8c). These results suggest that biological categories in modern and historic calculus samples cannot be directly compared, yet patterns of biological differences are maintained through time.

Similarly, no metabolites could be specifically attributed to bacterial processes, but several metabolites, including isovalerate, valerate, lactate, cadaverine and putrescene, are known metabolic products of oral bacteria (Scully et al. 1997; Takahashi 2015). Dipicolinate, which was detected in all modern and all historic samples, is the major component of bacterial spore capsules, and may indicate that bacterial endospore development occurs in mature plaque biofilms, or during plaque mineralization. Sulfate is abundant in GCF due to break-down of sulfur-containing amino acids by the oral biofilm, and is produced during anaerobic methanogenesis in oral plaque, however, we observed no correlation between the relative abundance of the oral methanogen Methanobrevibacter and sulfate levels (Figure S9) in our samples, and the very high abundance of sulfate in historic relative to modern calculus suggests an exogenous source. Oxford topsoil sulfur concentrations where the cemetery was located are in the 70th percentile across England and Wales (Rawlins et al. 2012), and sulfates in the soil and ground water of the cemetery may leach into the calculus by the same processes through which highly water-soluble metabolites are leached out of calculus. 
A

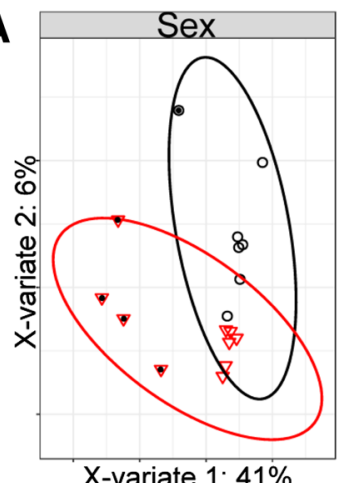

o Female - historic $\nabla$ Male - historic

O Female - modern

$\checkmark$ Male - modern

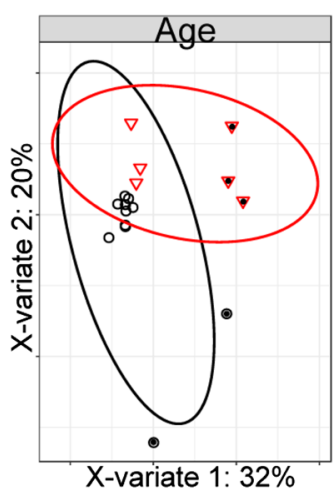

O 36+ - historic

$\nabla$ 18-35 - historic

○ 36+ - modern

$\nabla$ 18-35 - modern

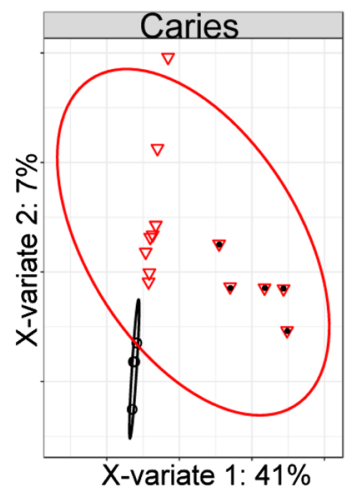

O No - historic

$\nabla$ Yes - historic

$\nabla$ Yes - modern

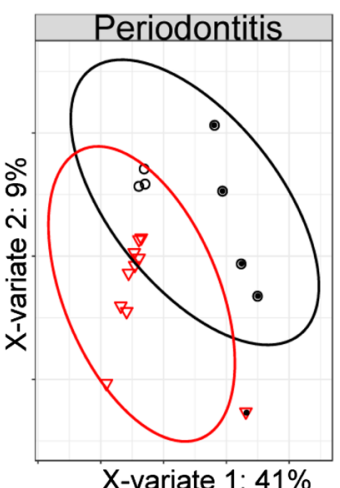

O No - historic

$\nabla$ Yes - historic

- No - modern

$\nabla$ Yes - modern

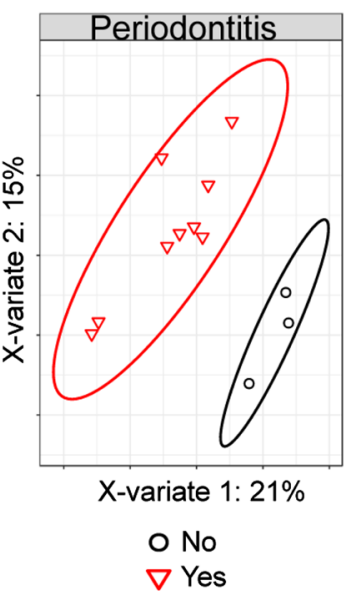

Fig. 5 Partial least squares discriminant analysis of metabolites detected at least one modern and one historic calculus sample. a Calculus samples cluster based on time period rather than biological category (sex, age, caries status and periodontal disease status) when including all metabolites detected in at least one modern and one his-

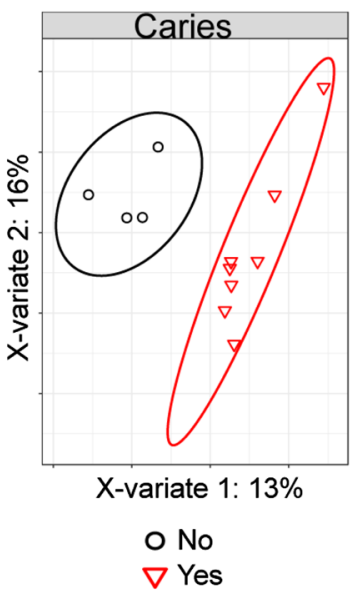

toric sample. b Historic calculus samples cluster based on biological category (sex, age, caries status and periodontal disease status) when including all metabolites detected in at least one historic sample. Ellipses indicate $95 \%$ confidence intervals

two by UW-Madison) increased the diversity of compounds we detected. However, unlike Dame et al. (2015), we found more metabolites by LC than GC methodologies, which was likely due to the low-abundance of molecules with higher aqueous solubility in the historic calculus that were analyzed by GC-MS. Although we found lower representation of aqueous-soluble molecules, in general calculus preserves a wide variety of molecules from the oral cavity and could be useful proxy for oral biofluids in archaeological samples. Calculus also provides an opportunity to co-investigate host and microbial activity, which is increasingly recognized as important to understanding cellular physiology and disease pathology (Takahashi 2015).

Saliva has been shown to preserve an individualized metabolic signature throughout daily routine (Wallner-Liebmann 
et al. 2016) and dental calculus, which contains salivary components, has the potential to preserve aspects of individual profiles over longer periods of time. While only five modern calculus samples were included in this study, PCA analysis revealed substantial metabolite profile diversity within these samples (Fig. 2a). It therefore appears that modern calculus may preserve individual phenotypes, although to investigate this, studies with larger sample sizes are needed to further assess this potential. Historic samples, in contrast, cluster much more tightly in the PCA, suggesting that individual phenotypes may be lost through metabolite degradation and loss over time. However, we were able to distinguish metabolic profile differences in historic samples between sex, age, and oral health status of the individuals by PLS-DA, demonstrating maintenance of individual profiles despite metabolite loss. It may then be possible to investigate differences in specific metabolite profile in historic samples, which could contribute to our understanding of disease demographics and evolution.

Relatively little is known about the process of agerelated protein degradation in archaeological samples, yet other historic samples provide some insight. Asparagine readily deamidates via cyclization to succinimidyl within chain; however, this mechanism is unavailable to the free amino acid. Under experimental heating, asparagine undergoes rapid hydrolysis (Crisp et al. 2013), and it is therefore probable that the free asparagine seen in the historic samples is derived from hydrolysis of peptides. Free glutamine and glutamic acid can undergo cyclisation to pyroglutamic acid even at low temperatures (Nagana Gowda et al. 2015). However, although pyroglutamate (pGlu; 5-oxoproline) is present at higher levels in the historic samples, it is too low to account for all the loss of all glutamic acid. This consistent pattern suggests that there is a contributing pool of degrading proteins, generating free amino acids which are undergoing modification, either in chain (e.g., asparagine deamidation) or once hydrolysed to terminal positions or as free amino acids (e.g., pyroglutamate). The majority of these low molecular weight, high solubility products are then likely lost from the calculus matrix. It is possible that some are so entrapped within the crystal matrix that they may persist as free amino acids and pyroglutamate, and this could be assessed by monitoring the level of racemization (Crisp et al. 2013).

Persistence of the Energy metabolites is unexpected given that most of the molecules in this category are small and water soluble, and therefore expected to be lost through time. Alpha-ketoglutarate may be produced by degradation of glutamate, which appears to be occurring in calculus as discussed in our results, and it may form stabilizing complexes with calcium, which is highly abundant in the predominantly calcium-phosphate mineral matrix of dental calculus. However, we speculate that these Energy metabolites may be fungal- or plant-derived compounds from the burial soil. Both plant roots and their associated mycorrhizal fungi secrete organic acids including citrate, malate, and oxalate (Badri and Vivanco 2009; Klugh and Cumming 2007), which are more abundant than expected in our historic samples. We expect that the endogenous Energy metabolites are lost over time through solubility, yet organic acids present in the soil from roots and fungi may diffuse into the historic calculus samples, and thus it appears that these metabolites are not lost through time. This concern may be addressed in future experiments by looking at preservation of small organic acids in historic calculus samples from individuals who were never buried in soil, such as from crypt burials, and from archaeological samples that have been in storage for varying periods of time.

Lipids, particularly unmodified, saturated classes, are some of the best-preserved metabolites in historic calculus, and appear to be particularly stable over time. Therefore, lipid analyses may be a promising focus for historic calculus studies. Although not a common focus in salivary or oral biofilm metabolomics studies, lipids are a versatile class of molecule with a broad range of physiological properties and actions. They play roles in local (intracellular) (Nishizuka 1995) and long-distance (hormone) cell signaling (Xavier et al. 2016), have both pro- and anti-inflammatory properties (Bennett and Gilroy 2016), and are the major components of cell membranes, where their composition influences cell membrane function (Zalba and ten Hagen 2017). Bacterial membrane lipid content varies by species (López-Lara and Geiger 2016), and may indicate bacterial physiological status (Darveau et al. 2004), while pathogenesis of the periodontal disease-associated oral bacteria Porphyromonas gingivalis is influenced by host cell membrane lipid composition (Wang and Hajishengallis 2008). The role of lipid mediators in the initiation and resolution of periodontal disease inflammation is currently under investigation (Bartold and Van Dyke 2013), and the wealth of lipids detected in calculus may be valuable to studies of both host and microbial pathophysiology.

Although we were unable to specifically identify bacterial contributions to the calculus metabolome, there are some metabolites suggestive of mature oral biofilm activity. Dipicolinate, which is the major capsule component of bacterial endospores, is a highly stable molecule, as evidenced by the long-term viability of endospores (Yung et al. 2007). To our knowledge, the inferred presence of endospores in calculus is a novel finding, as we were unable to find any references to the presence of bacterial endospores in oral plaque or dental calculus. Members of several Gram-positive genera that reside in the mouth have close relatives known to form spores, including Actinomyces (Gao and Gupta 2012), and Filifactor (Vos et al. 2011), and since many oral bacteria have not yet been genetically 
or physiologically characterized, it is possible that several oral species do have the ability to form spores. Calcification of the biofilm may induce a stress response in these species that initiates endospore formation, which would explain the abundance of dipicolinate in dental calculus.

Additionally, studies aiming to characterize salivary biomarkers of periodontal disease have identified several pathways with an apparent bacterial source that contain promising metabolite candidates for disease biomarkers (Kuboniwa et al. 2016; Sakanaka et al. 2017), and we have identified several of these in our calculus. Phenylalanine, succinate, hydrocinnimate, cadaverine, and putrescine are markers of periodontal disease that were reduced in saliva when supragingival plaque was removed, suggesting they were largely produced by bacteria (Sakanaka et al. 2017), and we detected each of these molecules. This demonstrates that bacterial metabolic products are present in calculus, and may offer insight into mature biofilm activity. This could be useful in studying how bacterial metabolism influences oral disease, as periodontal disease-associated oral plaque has community structure and activity much more similar to that of fully mature biofilms such as are found in calculus, than to healthy site subgingival plaque or supragingival plaque (Wade 2013), yet the presence of calculus alone is not a reflection of periodontal disease status (i.e., three of the five modern calculus samples were collected from teeth with no evidence of periodontal disease).

In sum, our results demonstrate that dental calculus contains an abundance of endogenous and exogenous metabolites, and that a wide range of these metabolites preserve well through time. Dental calculus therefore has significant potential to provide novel insights into human diet, physiology, and microbiome activity in both modern and historic samples, permitting human evolutionary and human-microbiome co-evolutionary studies with a deep-time perspective. Larger sample sizes and samples from additional temporal and cultural contexts as well as from varying burial and storage conditions are needed to further address metabolite preservation and presence/absence patterns. The excellent preservation of dental calculus in archaeological collections, however, means that there is ample opportunity to expand metabolite-based studies of dental calculus into the recent and distant past.

Acknowledgements Open access funding provided by Max Planck Society. The authors thank Mark Gibson, Tom Gilbert, Chris Gosden, Stuart Gould, Lauren McIntyre, Anita Radini, William Wade, and Helen Webb for assistance with sample and data collection. We would like to acknowledge The Danish National High-Throughput DNA Sequencing Centre for sequencing the samples. This work was supported by the Oxford University Fell Fund 143/108 (to G.L. and C.W.), the European Research Council ERC-2013-StG 337574-UNDEAD (to G.L.), the National Institutes of Health R01GM089886 (to C.M.L., C.W., and K.S.), the National Science Foundation BCS-1516633 (to
C.W. and C.M.L.), and BCS-1643318 (to C.W.), and R35GM118110 (to J.J.C.) and the National Library of Medicine T15LM007359 (to K.A.O.).

\section{Compliance with ethical standards}

Conflict of interest All authors declare that they have no conflict of interest.

Ethical approval The study was approved by the Institutional Review Board for Human Research Participant Protection at the University of Oklahoma [IRB\#4543] and was performed552 in accordance with the 1964 Helsinki declaration and its later amendments.

Informed consent Informed consent was obtained from all modern participants in the study prior to the collection of dental calculus

Open Access This article is distributed under the terms of the Creative Commons Attribution 4.0 International License (http://creativecommons.org/licenses/by/4.0/), which permits unrestricted use, distribution, and reproduction in any medium, provided you give appropriate credit to the original author(s) and the source, provide a link to the Creative Commons license, and indicate if changes were made.

\section{References}

Atkinson, W., Downer, P., Lever, M., Chambers, S. T., \& George, P. M. (2007). Effects of orange juice and proline betaine on glycine betaine and homocysteine in healthy male subjects. European Journal of Nutrition, 46(8), 446-452. doi:10.1007/ s00394-007-0684-5.

Badri, D. V., \& Vivanco, J. M. (2009). Regulation and function of root exudates. Plant, Cell \& Environment, 32(6), 666-681. doi:10.1111/j.1365-3040.2009.01926.x.

Barnes, V. M., Ciancio, S. G., Shibly, O., Xu, T., Devizio, W., Trivedi, H. M., et al. (2011). Metabolomics reveals elevated macromolecular degradation in periodontal disease. Journal of Dental Research, 90(11), 1293-1297. doi:10.1177/0022034511416240.

Barnes, V. M., Teles, R., Trivedi, H. M., Devizio, W., Xu, T., Mitchell, M. W., et al. (2009). Acceleration of purine degradation by periodontal diseases. Journal of Dental Research, 88(9), 851-855. doi: $10.1177 / 0022034509341967$.

Bartold, P. M., \& Van Dyke, T. E. (2013). Periodontitis: A host-mediated disruption of microbial homeostasis. Unlearning learned concepts. Periodontology 2000, 62, 203-217.

Beger, R. D. (2013). A review of applications of metabolomics in cancer. Metabolites, 3(3), 552-574. doi:10.3390/metabo3030552.

Bennett, M., \& Gilroy, D. W. (2016). Lipid mediators in inflammation. Microbiology Spectrum, 4(6). doi:10.1128/microbiolspec. MCHD-0035-2016.

Blatt, S. H., Redmond, B. G., Cassman, V., \& Sciulli, P. W. (2011). Dirty teeth and ancient trade: Evidence of cotton fibres in human dental calculus from Late Woodland, Ohio. International Journal of Osteoarchaeology, 21(6), 669-678. doi:10.1002/oa.1173.

Bouatra, S., Aziat, F., Mandal, R., Guo, A. C., Wilson, M. R., Knox, C., et al. (2013). The human urine metabolome. PLOS ONE, 8(9), e73076. doi:10.1371/journal.pone.0073076.

Brooks, S., \& Suchey, J. M. (1990). Skeletal age determination based on the os pubis: A comparison of the Acsádi-Nemeskéri and Suchey-Brooks methods. Human Evolution, 5(3), 227-238. doi:10.1007/BF02437238. 
Buckberry, J. L., \& Chamberlain, A. T. (2002). Age estimation from the auricular surface of the ilium: A revised method. American Journal of Physical Anthropology, 119(3), 231-239. doi:10.1002/ ajpa.10130.

Buckley, S., Usai, D., Jakob, T., Radini, A., \& Hardy, K. (2014). Dental calculus reveals unique insights into food items, cooking and plant processing in prehistoric Central Sudan. PLOS ONE, 9(7), e100808-e100810. doi:10.1371/journal.pone.0100808.

Caporaso, J. G., Kuczynski, J., Stombaugh, J., Bittinger, K., Bushman, F. D., Costello, E. K., et al. (2010). QIIME allows analysis of high-throughput community sequencing data. Nature Methods, 7(5), 335-336. doi:10.1038/nmeth.f.303.

Cappellini, E., Jensen, L. J., Szklarczyk, D., Ginolhac, A., da Fonseca, R. A. R., Stafford, T. W., et al. (2012). Proteomic analysis of a pleistocene mammoth femur reveals more than one hundred ancient bone proteins. Journal of Proteome Research, 11(2), 917-926. doi:10.1021/pr200721u.

Castillo-García, M. L., Aguilar-Caballos, M. P., \& Gómez-Hens, A. (2015). Determination of acetylsalicylic acid and its major metabolites in bovine urine using ultra performance liquid chromatography. Journal of Chromatography B, 985, 85-90. doi:10.1016/j. jchromb.2015.01.026.

Cosgrove, J. P., Church, D. F., \& Pryor, W. A. (1987). The kinetics of the autoxidation of polyunsaturated fatty acids. Lipids, 22(5), 299-304.

Crisp, M., Demarchi, B., Collins, M., Morgan-Williams, M., Pilgrim, E., \& Penkman, K. (2013). Isolation of the intra-crystalline proteins and kinetic studies in Struthio camelus (ostrich) eggshell for amino acid geochronology. Quaternary Geochronology, 16, 110-128. doi:10.1016/j.quageo.2012.09.002.

Dame, Z. T., Aziat, F., Mandal, R., Krishnamurthy, R., Bouatra, S., Borzouie, S., et al. (2015). The human saliva metabolome. Metabolomics, 11(6), 1864-1883. doi:10.1007/s11306-015-0840-5.

Darveau, R. P., Pham, T. T. T., Lemley, K., Reife, R. A., Bainbridge, B. W., Coats, S. R., et al. (2004). Porphyromonas gingivalis lipopolysaccharide contains multiple lipid a species that functionally interact with both toll-like receptors 2 and 4. Infection and Immunity, 72(9), 5041-5051. doi:10.1128/IAI.72.9.5041-5051.2004.

Daskalaki, E., Blackburn, G., Kalna, G., Zhang, T., Anthony, N., \& Watson, D. (2015). A study of the effects of exercise on the urinary metabolome using normalisation to individual metabolic output. Metabolites, 5(1), 119-139. doi:10.3390/metabo5010119.

Edgar, R. C. (2010). Search and clustering orders of magnitude faster than BLAST. Bioinformatics (Oxford, England), 26(19), 24602461. doi:10.1093/bioinformatics/btq461.

Ferembach, D., Schwindezky, I., \& Stoukal, M. (1980). Recommendation for age and sex diagnoses of skeletons. Journal of Human Evolution, 9, 517-549.

Fleet, J. C., Gliniak, C., Zhang, Z., Xue, Y., Smith, K. B., McCreedy, R., \& Adedokun, S. A. (2008). Serum metabolite profiles and target tissue gene expression define the effect of cholecalciferol intake on calcium metabolism in rats and mice. The Journal of Nutrition, 138(6), 1114-1120.

Foley, J. D., Sneed, J. D., Steinhubl, S. R., Kolasa, J., Ebersole, J. L., Lin, Y., et al. (2012). Oral fluids that detect cardiovascular disease biomarkers. Oral Surgery, Oral Medicine, Oral Pathology and Oral Radiology, 114(2), 207-214. doi:10.1016/j. oooo.2012.03.003.

Frantz, L. A. F., Mullin, V. E., Pionnier-Capitan, M., Lebrasseur, O., Ollivier, M., Perri, A., et al. (2016). Genomic and archaeological evidence suggest a dual origin of domestic dogs. Science, 352(6290), 1228-1231. doi:10.1126/science.aaf3161.

Gao, B., \& Gupta, R. S. (2012). Phylogenetic framework and molecular signatures for the main clades of the phylum Actinobacteria.
Microbiology and Molecular Biology Reviews, 76(1), 66-112. doi:10.1128/MMBR.05011-11.

Hahn, T. J., Hendin, B. A., Scharp, C. R., \& Haddad, J. G. (1972). Effect of chronic anticonvulsant therapy on serum 25-hydroxycalciferol levels in adults. The New England Journal of Medicine, 287(18), 900-904. doi:10.1056/NEJM197211022871803.

Hardy, K., Buckley, S., Collins, M. J., Estalrrich, A., Brothwell, D., Copeland, L., et al. (2012). Neanderthal medics? Evidence for food, cooking, and medicinal plants entrapped in dental calculus. Die Naturwissenschaften, 99(8), 617-626. doi:10.1007/ s00114-012-0942-0.

Hardy, K., Radini, A., Buckley, S., Sarig, R., Copeland, L., Gopher, A., \& Barkai, R. (2016). Dental calculus reveals potential respiratory irritants and ingestion of essential plant-based nutrients at lower Palaeolithic Qesem Cave Israel. Quaternary International, 398(c), 129-135. doi:10.1016/j.quaint.2015.04.033.

Hillson, S. (1996). Dental Anthropology. Cambridge: Cambridge University Press. doi:10.1017/CBO9781139170697.

Jensen, M. K., Bertoia, M. L., Cahill, L. E., Agarwal, I., Rimm, E. B., \& Mukamal, K. J. (2014). Novel metabolic biomarkers of cardiovascular disease. Nature Reviews Endocrinology, 10(11), 659-672. doi:10.1038/nrendo.2014.155.

Klugh, K. R., \& Cumming, J. R. (2007). Variations in organic acid exudation and aluminum resistance among arbuscular mycorrhizal species colonizing Liriodendron tulipifera. Tree Physiology, 27(8), 1103-1112. doi:10.1093/treephys/27.8.1103.

Knights, D., Kuczynski, J., Charlson, E. S., Zaneveld, J., Mozer, M. C., Collman, R. G., et al. (2011). Bayesian community-wide cultureindependent microbial source tracking. Nature Methods, 8(9), 761-763. doi:10.1038/nmeth.1650.

Kuboniwa, M., Sakanaka, A., Hashino, E., Bamba, T., Fukusaki, E., \& Amano, A. (2016). Prediction of periodontal inflammation via metabolic profiling of saliva. Journal of Dental Research, 95(12), 1381-1386. doi:10.1177/0022034516661142.

Lankinen, M., Kolehmainen, M., Jääskeläinen, T., Paananen, J., Joukamo, L., Kangas, A. J., et al. (2014). Effects of whole grain, fish and bilberries on serum metabolic profile and lipid transfer protein activities: A randomized trial (Sysdimet). PLoS ONE, 9(2), e90352. doi:10.1371/journal.pone.0090352.

Lever, M., Sizeland, P. C. B., Bason, L. M., Hayman, C. M., \& Chambers, S. T. (1994). Glycine betine and proline betaine in human blood and urine. Biochimica et biophysica acta, 1200, 259-264.

Lovejoy, C. O., Meindl, R. S., Pryzbeck, T. R., \& Mensforth, R. P. (1985). Chronological metamorphosis of the auricular surface of the ilium: A new method for the determination of adult skeletal age at death. American Journal of Physical Anthropology, 68(1), 15-28. doi:10.1002/ajpa.1330680103.

López-Lara, I. M., \& Geiger, O. (2016). Bacterial lipid diversity. Biochimica et Biophysica Acta. doi:10.1016/j.bbalip.2016.10.007.

Meyer, M., Kircher, M., Gansauge, M.-T., Li, H., Racimo, F., Mallick, S., et al. (2012). A high-coverage genome sequence from an archaic Denisovan individual. Science, 338(6104), 222-226. doi:10.1126/science. 1224344 .

Nagana Gowda, G. A., Gowda, Y. N., \& Raftery, D. (2015). Massive glutamine cyclization to pyroglutamic acid in human serum discovered using NMR spectroscopy. Analytical chemistry, 87(7), 3800-3805. doi:10.1021/ac504435b.

Nishizuka, Y. (1995). Protein kinase C and lipid signaling for sustained cellular responses. The FASEB Journal, 9(7), 484-496. doi:10.1096/fj.1530-6860.

Oakley, L. H., Casadio, F., Shull, K. R., \& Broadbelt, L. J. (2015). Microkinetic modeling of the autoxidative curing of an alkyd and oil-based paint model system. Applied Physics A, 121(3), 869-878. doi:10.1007/s00339-015-9363-1. 
Ogden, A. (2005). A new and simple system for the recording of periodontal disease in skeletal material (pp. 1-1). Presented at the British Association for Biological Anthropology and Osteoarchaeology.

Parks, D. H., \& Beiko, R. G. (2010). Identifying biologically relevant differences between metagenomic communities. Bioinformatics (Oxford, England), 26(6), 715-721. doi:10.1093/bioinformatics/ btq041.

Parks, D. H., Tyson, G. W., Hugenholtz, P., \& Beiko, R. G. (2014). STAMP: Statistical analysis of taxonomic and functional profiles. Bioinformatics (Oxford, England), 30(21), 3123-3124. doi:10.1093/bioinformatics/btu494.

Phenice, T. W. (1969). A newly developed visual method of sexing the os pubis. American Journal of Physical Anthropology, 30(2), 297-301. doi:10.1002/ajpa.1330300214.

Psychogios, N., Hau, D. D., Peng, J., Guo, A. C., Mandal, R., Bouatra, S., et al. (2011). The human serum metabolome. PLoS ONE, 6(2), e16957. doi:10.1371/journal.pone.0016957.

Radini, A., Buckley, S., Rosas, A., Estalrrich, A., la Rasilla, de, M., \& Hardy, K. (2016). Neanderthals, trees and dental calculus: New evidence from El Sidró n. Antiquity, 90(350), 290-301. doi:10.15184/aqy.2016.21.

Rawlins, B. G., McGrath, S. P., Scheib, A., Breward, N., Cave, M. R., Lister, B., et al. (2012). The advanced soil geochemical atlas of England and Wales. Nottingham: British Geological Survey.

Rohart, F., Gautier, B., Singh, A., \& Le Cao, K.-A. (2017). mixOmics (draft1): An R package for 'omics feature selection and multiple data integration. bioRxiv, 108597. doi:10.1101/108597.

Rustan, A. C., \& Drevon, C. A. (2005). Fatty acids: Structures and properties. Encyclopedia of life sciences. Wiley. doi:10.1038/ npg.els.0003894.

Sakanaka, A., Kuboniwa, M., Hashino, E., Bamba, T., Fukusaki, E., \& Amano, A. (2017). Distinct signatures of dental plaque metabolic byproducts dictated by periodontal inflammatory status. Scientific Reports, 7, 42818. doi:10.1038/srep42818.

Schwartz, J. H. (1996). Skeleton keys: An introduction to human skeletal morphology, development, and analysis. Oxford Univeristy Press. doi:10.1007/BF02735270.

Scully, C., el-Maaytah, M., Porter, S. R., \& Greenman, J. (1997). Breath odor: Etiopathogenesis, assessment and management. European Journal of Oral Sciences, 105(4), 287-293.

Skoglund, P., Ersmark, E., Palkopoulou, E., \& Dalén, L. (2015). Ancient wolf genome reveals an early divergence of domestic dog ancestors and admixture into high-latitude breeds. Current Biology: CB, 25(11), 1515-1519. doi:10.1016/j. cub.2015.04.019.

Skoglund, P., Storå, J., Götherström, A., \& Jakobsson, M. (2013). Accurate sex identification of ancient human remains using DNA shotgun sequencing. Journal of Archaeological Science, 40(12), 4477-4482. doi:10.1016/j.jas.2013.07.004.

Sugimoto, M., Wong, D. T., Hirayama, A., Soga, T., \& Tomita, M. (2010). Capillary electrophoresis mass spectrometry-based saliva metabolomics identified oral, breast and pancreatic cancer-specific profiles. Metabolomics, 6(1), 78-95. doi:10.1007/ s11306-009-0178-y.

Sysi-Aho, M., Ermolov, A., Gopalacharyulu, P. V., Tripathi, A., Seppänen-Laakso, T., Maukonen, J., et al. (2011). Metabolic regulation in progression to autoimmune diabetes. PLoS Computational Biology, 7(10), e1002257. doi:10.1371/journal. pcbi. 1002257.

Takahashi, N. (2015). Oral microbiome metabolism: From "Who are they?" to "What are they doing?". Journal of Dental Research ,94(12), 16285-1637

Takahashi, N., Washio, J., \& Mayanagi, G. (2010). Metabolomics of supragingival plaque and oral bacteria. Journal of Dental Research, 89(12), 1383-1388. doi:10.1177/0022034510377792.
Takeda, I., Stretch, C., Barnaby, P., Bhatnager, K., Rankin, K., Fu, H., et al. (2009). Understanding the human salivary metabolome. NMR in Biomedicine, 22(6), 577-584. doi:10.1002/ nbm. 1369 .

Tetko, I. V., \& Bruneau, P. (2004). Application of ALOGPS to predict 1-octanol/water distribution coefficients, $\log \mathrm{P}$, and $\log \mathrm{D}$, of AstraZeneca in-house database. Journal of Pharmaceutical Sciences, 93(12), 3103-3110. doi:10.1002/jps.20217.

Velasco, J., \& Dobarganes, C. (2002). Oxidative stability of virgin olive oil. European Journal of Lipid Science and Technology, 104(9-10), 661-676. doi:10.1002/1438-9312(200210)104:9/10<661

Vos, P., Garrity, G., Jones, D., Krieg, N. R., Ludwig, W., Rainey, F. A., et al. (2011). Bergey's manual of systematic bacteriology.

Wade, W. G. (2013). The oral microbiome in health and disease. Pharmacological Research, 69(1), 137-143. doi:10.1016/j. phrs.2012.11.006.

Wallner-Liebmann, S., Tenori, L., Mazzoleni, A., Dieber-Rotheneder, M., Konrad, M., Hofmann, P., et al. (2016). Individual human metabolic phenotype analyzed by $1 \mathrm{H}$ NMR of saliva samples. Journal of Proteome Research, 15, 1787-1793. doi:10.1021/acs.jproteome.5b01060

Wang, Mingxun, Carver, J. J., Phelan, V. V., Sanchez, L. M., Garg, N., Peng, Y., et al. (2016). Sharing and community curation of mass spectrometry data with global natural products social molecular networking. Nature Biotechnology, 34(8), 828-837. doi:10.1038/nbt.3597.

Wang, Min, \& Hajishengallis, G. (2008). Lipid raft-dependent uptake, signalling and intracellular fate of Porphyromonas gingivalis in mouse macrophages. Cellular Microbiology, 10(10), 2029-2042. doi:10.1111/j.1462-5822.2008.01185.x.

Warinner, C. (2016). Dental Calculus and the evolution of the human oral microbiome. Journal of the California Dental Association, 44(7), 411-420.

Warinner, C., Hendy, J., Speller, C., Cappellini, E., Fischer, R., Trachsel, C., et al. (2014). Direct evidence of milk consumption from ancient human dental calculus. Scientific Reports, 4, 7104. doi:10.1038/srep07104.

Warinner, C., Rodrigues, J. F. M., Vyas, R., Trachsel, C., Shved, N., Grossmann, J., et al. (2014). Pathogens and host immunity in the ancient human oral cavity. Nature Genetics, 46(4), 336-344. doi:10.1038/ng.2906.

Warinner, C., Speller, C., \& Collins, M. J. (2015). A new era in palaeomicrobiology: Prospects for ancient dental calculus as a long-term record of the human oral microbiome. Philosophical Transactions of the Royal Society B, 370, 20130376.

White, D. J. (1991). Processes contributing to the formation of dental calculus. Biofouling, 4(1-3), 209-218. doi:10.1080/08927019109378211.

Wishart, D. S., Jewison, T., Guo, A. C., Wilson, M., Knox, C., Liu, Y., et al. (2013). HMDB 3.0-The human metabolome database in 2013. Nucleic Acids Research, 41(D1), D801-D807. doi:10.1093/nar/gks1065.

Xavier, A. M., Anunciato, A. K. O., Rosenstock, T. R., \& Glezer, I. (2016). Gene expression control by glucocorticoid receptors during innate immune responses. Frontiers in Endocrinology, 7, 31. doi:10.3389/fendo.2016.00031.

Xia, Y.-Q., Patel, S., Bakhtiar, R., Franklin, R. B., \& Doss, G. A. (2005). Identification of a new source of interference leached from polypropylene tubes in mass-selective analysis. Journal of the American Society for Mass Spectrometry, 16(3), 417-421. doi:10.1016/j.jasms.2004.11.020.

Yamada, T., Letunic, I., Okuda, S., Kanehisa, M., \& Bork, P. (2011). iPath2.0: Interactive pathway explorer. Nucleic Acids Research, 39(suppl), W412-W415. doi:10.1093/nar/gkr313. 
Yan, S.-K., Wei, B.-J., Lin, Z.-Y., Yang, Y., Zhou, Z.-T., \& Zhang, W.-D. (2008). A metabonomic approach to the diagnosis of oral squamous cell carcinoma, oral lichen planus and oral leukoplakia. Oral Oncology, 44(5), 477-483. doi:10.1016/j. oraloncology.2007.06.007.

Yung, P. T., Shafaat, H. S., Connon, S. A., \& Ponce, A. (2007). Quantification of viable endospores from a Greenland ice core. FEMS Microbiology Ecology, 59(2), 300-306. doi:10.1111/j.1574-6941.2006.00218.x.

Zalba, S., \& ten Hagen, T. L. M. (2017). Cell membrane modulation as adjuvant in cancer therapy. Cancer Treatment Reviews, 52, 48-57. doi:10.1016/j.ctrv.2016.10.008.
Zhang, A., Sun, H., \& Wang, X. (2012). Saliva metabolomics opens door to biomarker discovery, disease diagnosis, and treatment. Applied Biochemistry and Biotechnology, 168(6), 1718-1727. doi:10.1007/s12010-012-9891-5.

Ziesemer, K. A., Mann, A. E., Sankaranarayanan, K., Schroeder, H., Ozga, A. T., Brandt, B. W., et al. (2015). Intrinsic challenges in ancient microbiome reconstruction using 16S rRNA gene amplification. Scientific Reports, 5, 16498-16419. doi:10.1038/ srep16498. 